\title{
Adaptation Strategies for Applying AWGN-Based Denoiser to Realistic Noise
}

\author{
Yuqian Zhou, ${ }^{\dagger}$ Jianbo Jiao, ${ }^{\dagger}$ Haibin Huang, ${ }^{\ddagger}$ Jue Wang, ${ }^{\ddagger}$ Thomas Huang ${ }^{\dagger}$ \\ ${ }^{\dagger}$ Beckman Institute, University of Illinois at Urbana-Champaign, Urbana, IL 61801, USA \\ ${ }^{\ddagger}$ Megvii (Face++), Redmond, WA 98052, USA \\ zhouyuqian133@gmail.com
}

\begin{abstract}
Discriminative learning based denoising model trained with Additive White Gaussian Noise (AWGN) performs well on synthesized noise. However, realistic noise can be spatialvariant, signal-dependent and a mixture of complicated noises. In this paper, we explore multiple strategies for applying an AWGN-based denoiser to realistic noise. Specifically, we trained a deep network integrating noise estimating and denoiser with mixed Gaussian (AWGN) and Random Value Impulse Noise (RVIN). To adapt the model to realistic noises, we investigated multi-channel, multi-scale and super-resolution approaches. Our preliminary results demonstrated the effectiveness of the newly-proposed noise model and adaptation strategies.
\end{abstract}

\section{Introduction}

Discriminative learning based methods like DnCNN(Zhang et al. 2017) have achieved state-of-the-art performance on image blind denoising. However, there are some problems of the black-box methods, including lacking the flexibility of noise level and type changes, and not working on spatialvariant noise. Always trained on paired synthesized data, it lacks the capability of denoising realistic noises.

In this work, we proposed an image blind denoising method by concatenating an explicit noise estimator and an adaptive denoiser. To improve the generalization of the system, we considered AWGN and RVIN as our basic noise types. The estimator will be used to estimate the level of different noises across RGB channels, and the estimated level with the noisy image will be fed in to the denoiser. Specifically, we use the maps of the same size as the image to represent pixel-wise noise levels, thus the model is efficient in processing spacial-variant and type-variant noise, even mixture of noises. We investigated the difference between synthesized noise and real noise, and utilized multi-scale, multi-channel and super-resolution strategies to improve the model robustness for real noise. Our results demonstrate that our model is more powerful in processing mixed noise and real noise.

Copyright (C) 2019, Association for the Advancement of Artificial Intelligence (www.aaai.org). All rights reserved.

\section{Proposed Model}

The model consists of a noise estimator $N_{e s}$ and an adaptive non-blind denoiser $R_{e s}$. Noise levels $N_{e s}\left(x_{n}\right)$ are represented by six noise level maps corresponding to levels of AWGN and RVIN across three channels. Then the noisy images $x_{n}$ are concatenated with the estimated noise maps $N_{e s}\left(x_{n}\right)$ and input to the denoiser $R_{e s}$ which is a 20-layer DnCNN. The model will output a residual $R_{e s}\left(x_{n}, N_{e s}\left(x_{n}\right)\right)$ to be matched with the true noise $r_{t r}$. In addition to the estimated noise maps, the true levels $n_{t r}$ are also concatenated to the noisy image $x_{n}$ for residual learning. This is used to make the denoiser work for both non-blind and blind denoising. The full objectives then can be represented as,

$$
\begin{aligned}
L= & \alpha L_{r e s}\left(r_{t r}, R_{e s}\left(x_{n}, N_{e s}\left(x_{n}\right)\right)\right. \\
& +\beta L_{n l}\left(n_{t r}, N_{e s}\left(x_{n}\right)\right)+\gamma L_{g r e s}\left(r_{t r}, R_{e s}\left(x_{n}, n_{t r}\right)\right),
\end{aligned}
$$

here all the $L$ are 12-norm, and $\alpha, \beta$, and $\gamma$ are set to be equal in our experiment.

For each type of noise, we set up a pre-defined noise range. Specifically, for AWGN, the range of the $\sigma$ is from 0 to 75 . For RVIN, the levels are represented by the mix ratio $r_{k}$ which are mostly 0.3 for channel $k$. To cope with mixed noise types, we also synthesize mixed AWGN with RVIN by,

$$
n_{k}(i, j)= \begin{cases}\text { uniform }(0,255), & \text { if } p_{k}(i, j)<r_{k} \\ x(i, j, k)+A W G N\left(0, s t d_{k}\right), & \text { otherwise }\end{cases}
$$

where $p$ is the random map following uniform distribution from 0 to 1 . All the levels are normalized to $[0,1]$, and will be clamped and refined before inputting to the denoiser.

\section{Experiments}

We followed the training data sampling of DnCNN(Zhang et al. 2017) to crop $50 \times 50$ patches from 500 color images of size $180 \times 180$. For testing dataset, we utilized the benchmark data from the widely used 20 images as in PGB (Xu et al. 2016). For real noise, we tested it on DND Benchmark(Plötz and Roth 2017). We evaluated the performance of the algorithm in terms of PSNR and SSIM, as well as the visual quality.

\section{Mixed Synthesized AWGN and RVIN Noise}

We evaluated our model on eliminating mixed AWGN and RVIN as in PGB (Xu et al. 2016). We compared our methods 

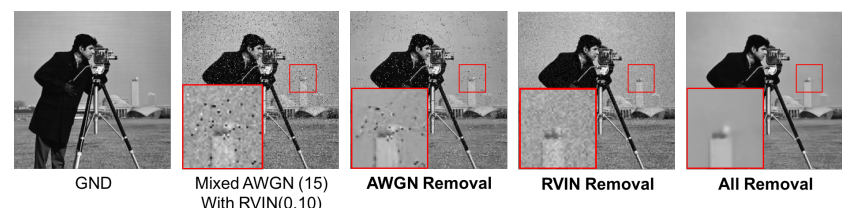

Figure 1: AWGN and RVIN disentangling.
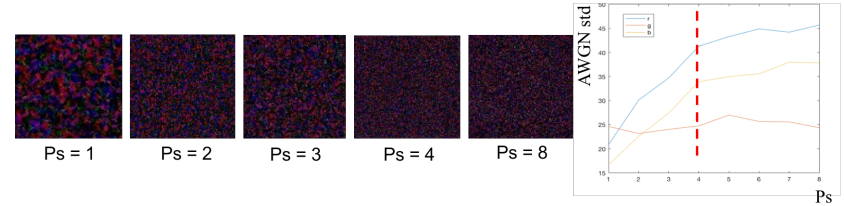

Figure 2: Realistic Noise Estimation after pixel-shuffle subsampling with MC-AWGN-RVIN Model. The estimated AWGN level for RGB channel will keep steady after about stride 4 in this real noisy image. Ps: Pixel-shuffle stride

with most previous work listed in (Xu et al. 2016) including non-blind Gaussian denoiser BM3D (Dabov et al. 2006), and models desingned for mixed AWGN and RVIN like WESNR (Jiang, Zhang, and Yang 2014). The results are shown in Table 1. Our model achieved the best performance. The proposed model can also be used to eliminate different types of noises separately. As shown in Figure 1, after we zero out the unrelated channels, we are able to denoise only AWGN or RVIN without influencing the other.

\begin{tabular}{l|c|c|c|c|c}
\hline$\sigma, r$ & BM3D & WESNR & PGB & DnCNN-B & Ours \\
\hline $10,0.15$ & 25.18 & 25.41 & 27.17 & 32.09 & $\mathbf{3 2 . 3 3}$ \\
$10,0.30$ & 21.80 & 21.40 & 22.17 & 29.97 & $\mathbf{3 0 . 3 5}$ \\
$20,0.15$ & 25.13 & 23.57 & 26.12 & 29.52 & $\mathbf{2 9 . 7 4}$ \\
$20,0.30$ & 21.73 & 21.40 & 21.89 & 27.90 & $\mathbf{2 8 . 2 1}$ \\
\hline
\end{tabular}

Table 1: Comparison of PSNR results on mixture of AWGN and RVIN removal performance.

\section{Multi-channel and Multi-scale Strategy}

To adapt AWGN-RVIN-based noise model to real noise, we estimated each channel separately and applied pixel-shuffle for subsampling before denoising. Pixel-shuffle is used to select pixels by strides and combine the sub figures into a mosaic. To choose the appropriate stride, we utilized the trained AWGN estimator to select the optimal one. As shown in Figure 2, as we increase the stride, the low-frequency realistic noise will become more similar to AWGN in patterns, and the estimated noise level will first increase and then keep steady. We kept the stride number which makes the downscaled realistic noise just suitable for the AWGN noise model as the optimal one to denoise.

\section{Super-resolution Strategy}

The main problem of current denoising models is oversmoothing and losses of textural details. The blur caused by
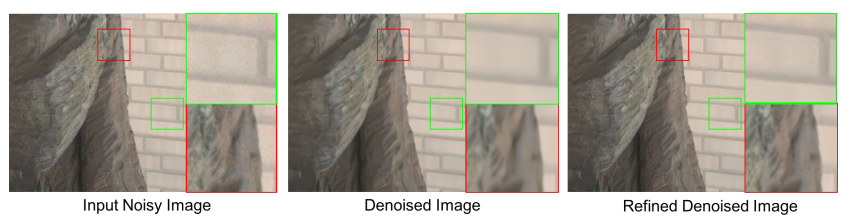

Figure 3: Denoising results on DND dataset with or without super-resolution refinement.

denoising algorithms can be treated as a special type of image degradation, thus we implemented an external five-layer CNN as the refinement network to add details and improve visual quality by taking the over-smoothed denoised images as inputs. Some examples of the refinement are shown in Figure 3.

\section{Results on DND Benchmark}

With the strategies mentioned above, we evaluated the model on DND benchmark and compare it with other models which are also based on AWGN. Our model achieved better performance as shown in Table 2. For the super-resolution refinement, even though the numerical metrics are lower, better visual quality is achieved as shown in Figure 3.

\begin{tabular}{c|c|c|c|c|c|c}
\hline & \multicolumn{3}{|c|}{ non-blind } & \multicolumn{3}{c}{ blind } \\
\hline & BM3D & WNNM & Ours & DnCNN & Ours(s) & Ours \\
\hline PSNR & 34.51 & 34.67 & $\mathbf{3 7 . 2 0}$ & 32.43 & 35.72 & $\mathbf{3 7 . 1 2}$ \\
SSIM & 0.8507 & 0.8646 & $\mathbf{0 . 9 3 6 1}$ & 0.7900 & 0.9086 & $\mathbf{0 . 9 3 5 3}$ \\
\hline
\end{tabular}

Table 2: Comparison of PSNR and SSIM on DND Benchmark. ours(s): models with super-resoulution refinement.

\section{References}

Dabov, K.; Foi, A.; Katkovnik, V.; and Egiazarian, K. 2006. Image denoising with block-matching and $3 \mathrm{~d}$ filtering. In Image Processing: Algorithms and Systems, Neural Networks, and Machine Learning, volume 6064, 606414. International Society for Optics and Photonics.

Jiang, J.; Zhang, L.; and Yang, J. 2014. Mixed noise removal by weighted encoding with sparse nonlocal regularization. IEEE transactions on image processing 23(6):2651-2662.

Plötz, T., and Roth, S. 2017. Benchmarking denoising algorithms with real photographs. In $C V P R$, volume 1, 6 .

Xu, J.; Ren, D.; Zhang, L.; and Zhang, D. 2016. Patch group based bayesian learning for blind image denoising. In Asian Conference on Computer Vision, 79-95. Springer.

Zhang, K.; Zuo, W.; Chen, Y.; Meng, D.; and Zhang, L. 2017. Beyond a gaussian denoiser: Residual learning of deep cnn for image denoising. IEEE Transactions on Image Processing 26(7):3142-3155. 